\title{
A longitudinal twin study of the association between childhood autistic traits and psychotic experiences in adolescence
}

\author{
Mark J. Taylor ${ }^{1}$, Elise B. Robinson ${ }^{2,3}$, Francesca Happé ${ }^{4}$, Patrick Bolton ${ }^{5}$, Daniel Freeman ${ }^{6}$ and Angelica Ronald ${ }^{1 *}$
}

\begin{abstract}
Background: This twin study investigated whether autistic traits during childhood were associated with adolescent psychotic experiences.

Methods: Data were collected from a community sample of approximately 5000 twin pairs, which included 32 individuals with diagnosed autism spectrum conditions (ASC). Parents rated autistic traits in the twins at four points between ages 8-16 years. Positive, negative, and cognitive psychotic experiences were assessed at age 16 years using self- and parent-report scales. Longitudinal twin analyses tested the associations between these measures.

Results: Autistic traits correlated weakly or nonsignificantly with positive psychotic experiences (paranoia, hallucinations, and grandiosity), and modestly with cognitive psychotic experiences (cognitive disorganisation). Higher correlations were observed for parent-rated negative symptoms and self-reported anhedonia, although the proportion of variance in both accounted for by autistic traits was low (10 and $31 \%$, respectively). The majority of the genetic influences on negative symptoms and anhedonia were independent of autistic traits. Additionally, individuals with ASC displayed significantly more negative symptoms, anhedonia, and cognitive disorganisation than controls.

Conclusions: Autistic traits do not appear to be strongly associated with psychotic experiences in adolescence; associations were also largely restricted to negative symptoms. Of note, the degree to which the genetic and environmental causes of autistic traits influenced psychotic experiences was limited. These findings thus support a phenotypic and etiological distinction between autistic traits and psychotic experiences.
\end{abstract}

Keywords: Autism, Psychotic experiences, Twin study, Genetics, Comorbidity

\section{Background}

Autism spectrum conditions (ASC) are characterised by atypical social communication and behavioural inflexibility [1]. While historically viewed as childhood schizophrenia, evidence on psychotic disorders in individuals with ASC is mixed. While some studies report a very low prevalence of psychotic disorders amongst individuals with ASC [2-4], others have reported prevalence as high as $13 \%$ [5].

Psychotic experiences are milder manifestations of psychotic symptoms that commonly present in adolescence

\footnotetext{
* Correspondence: a.ronald@bbk.ac.uk

${ }^{1}$ Centre for Brain and Cognitive Development, Department of Psychological

Sciences, Birkbeck, University of London, London, UK

Full list of author information is available at the end of the article
}

[6-9]. While evidence on clinical psychotic disorders in individuals with ASC is inconsistent, psychotic experiences appear relatively commonly in individuals with ASC $[10$, 11]. In two studies, negative symptoms were elevated in young children with ASC $[12,13]$. In another, $5.4 \%$ of children with ASC reported hallucinations [14]. Thus, the findings to date suggest that particular psychotic experiences may present relatively frequently in children with ASC.

Psychotic experiences are associated with elevated distress, suicide risk, and subsequent psychotic disorders [15-17], and so, there is a need to understand why they co-occur with ASC. There is some indication of overlapping causes between clinical psychotic disorders and ASC. ASC and schizophrenia seem to run together in the same families [18], and there is tentative evidence that certain 
genes may link with both conditions [19]. The complete picture is not clear, however; certain copy-number variants are unique to each condition, while a low genetic correlation, calculated using common SNPs, has been reported (.16) [19, 20].

It is also unknown whether the association between ASC and psychotic experiences is due to shared causes. Psychotic experiences display modest to moderate heritability across differing severity levels [21]. Likewise, subclinical autistic traits are highly heritable, again consistently across different severity levels [22]. If there is genetic overlap between clinical forms of these traits, one might hypothesise that the causes of autistic traits and psychotic experiences will also overlap. That is, one might predict that the genetic variants underlying autistic traits will also influence continuous variation in psychotic experiences in the general population.

The twin design can address this question, as it allows estimation of the extent of overlap in genetic and environmental influences between two traits. Prior studies of ASC and psychotic experiences have employed data from singletons [10-14], meaning that they were unable to test the degree of genetic and environmental overlap across these phenotypes. We therefore aimed to investigate the degree to which the causes of autistic traits across an 8-year period of childhood would account for adolescent psychotic experiences. Psychotic experiences comprise multiple factors $[8,23]$; hence, we focused on individual psychotic experiences in adolescence. We also examined whether individuals with ASC displayed more psychotic experiences than typically developing individuals.

We expected genetic influences on autistic traits to account for a significant degree of variance in adolescent psychotic experiences, specifically negative symptoms $[13,14]$. Individuals with ASC were expected to display more negative symptoms, in particular, than typically developing individuals.

\section{Methods}

\section{Participants}

Participants were taking part in the Twins Early Development Study (TEDS), a community sample of twins born in England and Wales between 1994 and 1996 [24]. When twins were aged 16, 10,874 families were invited to take part in the Longitudinal Experiences And Perceptions (LEAP) study, a study of psychotic experiences in the general population. In total, 5128 parents returned LEAP data, while 5074 twin pairs both returned questionnaires. Participating and nonparticipating families in LEAP were similar on various demographic characteristics (shown in Additional file 1). Autistic traits were assessed four times: at ages 8,12, 14, and 16. All participants in LEAP who had autistic trait data at any prior age were included in the study. Exclusions were conducted for genetic syndromes, chromosomal abnormalities, perinatal/postnatal complications, and missing first contact or zygosity data. The final sample sizes are shown in Table 1. Zygosity was determined through parent report of twin resemblance and DNA testing [25]. TEDS has full ethical approval from the King's College London, Institute of Psychiatry Ethics Committee. All participants provided informed consent before completing questionnaires.

\section{Measures \\ Autistic traits}

Autistic traits were assessed at all four ages. At ages 8 and 12 years, parents completed the Childhood Autism Spectrum Test (CAST [26]), a 31-item questionnaire (30 items were used at age 12 years due to the removal of an age-inappropriate item). Each question was answered 'yes' or 'no'; possible ranges of scores are shown in Table 1. The measure was reliable (see Table 1) and stable between ages 8 and 12 years $(r=.55, p<.001)$. Scores over 15 have $100 \%$ sensitivity and $97 \%$ specificity in detecting individuals at risk of ASC, with a positive predictive value of $50 \%$ [27]. The CAST was divided into three previously published subscales [28]: social difficulties, communication atypicalities, and repetitive behaviours and interests.

The CAST is a measure for elementary school-aged children [26]; hence, parents completed the shortened Autism Spectrum Quotient (AQ [29, 30]) at ages 14 and 16 years. The AQ comprised 38 items at age 14 years and 28 items at age 16 years. Parents rated their agreement with each item on a 4-point scale. The AQ was reliable (see Table 1) and stable between ages 14 and 16 years $(r=.67, p<.001)$. Individuals with ASC scored significantly higher on the AQ at ages $14\left(t_{2546}=-9.43\right.$,

Table 1 Interpretation of twin correlations

\begin{tabular}{lll}
\hline Component & Cross-twin correlation & Cross-trait cross-twin correlation \\
\hline Additive genetic (A) & MZ correlation is higher than the DZ correlation & MZ correlation is higher than the DZ correlation \\
Shared environmental (C) & DZ correlation is greater than half the MZ correlation & DZ correlation is greater than half the MZ correlation \\
Nonshared environmental (E) & MZ correlation is lower than 1 & MZ correlation is lower than the phenotypic correlation
\end{tabular}

Cross-twin correlation: correlation of one twin's score on a trait with their co-twin's score on the same trait; cross-trait cross-twin correlation: correlation of one twin's score on a trait with their co-twin's score on another

$M Z$ monozygotic twins, DZ dizygotic twins 
$p<.001)$ and 16 years $\left(t_{4894}=-15.00, p<.001\right)$. The AQ was divided into subscales [29]: social difficulties, communication, imagination, attention to detail, and attention switching. Communication items were omitted from the scale at age 16 owing to space constraints.

\section{ASC diagnoses}

Parents of twins scoring over 15 on the CAST were invited to complete the Diagnostic and Well-Being Assessment (DAWBA [31]), a structured interview for establishing psychiatric diagnoses. Thirty-eight ASCrelevant items were administered over the telephone. The DAWBA effectively distinguishes between ASC cases and controls ${ }^{30}$, and has good inter-rater reliability $(\kappa=0.83)$ and internal consistency $(\alpha=0.92)$ in this sample [32]. If either twin in a pair met DAWBA ASC criteria, families were invited to take part in the Social Relationships Study [33]. Two trained researchers visited the families to complete the Autism Diagnostic Observation Schedule [34] with the twins and Autism Diagnostic Interview-Revised [35] with the parents; these semistructured assessments are considered the 'gold standard' in diagnosing ASC in research. In total, 32 participants with a confirmed diagnosis had Specific Psychotic Experiences Questionnaire (SPEQ) data available (29 males, 3 females).

\section{Psychotic experiences}

Psychotic experiences at age 16 were assessed using the SPEQ [8]. The SPEQ was constructed from six existing measures of psychotic experiences in adults. The wording of these measures was adapted for use with adolescents, and the age appropriateness of the items was established in terms of expert clinical opinion. Positive and cognitive psychotic experiences were assessed using four self-report measures: paranoia (15 items), hallucinations (9 items), cognitive disorganisation (11 items), and grandiosity (8 items). Negative symptoms were assessed via a self-report measure of anhedonia (10 items) and a parent measure of negative symptoms ( 9 items). The use of both a parent- and self-report measure of negative symptoms was designed to reflect recent recommendations that assessment of negative symptoms should include multiple informants [36]. One item ('Has few or no friends') was removed from negative symptoms owing to overlap with the autistic trait measures. Table 1 shows ranges of scores and internal consistencies. All subscales were stable across a 9-month period $(r=.65-.74)$. Principal-components analysis supported the division of SPEQ into six subscales [8]. The measure has been validated against a similar measure, the Psychosis-Like Symptoms (PLIKS) scale $[8,37]$. Individuals reporting having 'definitely' had psychotic experiences on the PLIKS scored significantly higher on all SPEQ subscales than those who did not (all $p<.001$ ). Individuals with relatives with schizophrenia or bipolar disorder $(N=420,227$ males, 193 females) scored significantly higher on all SPEQ subscales $(p<.05)$, except for anhedonia and hallucinations (which showed a nonsignificant trend).

\section{Data analyses}

Skewed measures were log transformed (Table 1). Sex and age effects can inflate twin correlations (described below). As a result, all scores were regressed for sex and age, as is standard behavioural genetic procedure [38]. Two sets of analyses were performed. Multivariate twin models were fitted to data on continuous autistic traits and psychotic experiences in the whole sample. Mean group differences in continuous SPEQ scores were then compared in individuals with a diagnosis of ASC and those without such a diagnosis.

Analyses were performed in the OpenMx [39] package of R [40]. OpenMx uses full information maximum likelihood when fitting models, meaning that missing data can be accounted for. Rather than excluding participants with any missing data, full information maximum likelihood includes participants with data for at least one age, meaning that the sample is not limited by the presence of missing data [39].

\section{Phenotypic analyses}

Phenotypic correlations $\left(r_{\mathrm{ph}}\right)$ between measures were estimated from twin models.

Mean standardised SPEQ scores were compared across individuals with ASC and controls using independent $t$ tests. In addition, we tested whether individuals with a family history of a psychotic disorder would display more autistic traits than those without such a family history, again using independent $t$ tests. Welch's degrees of freedom for unequal sample sizes were applied.

\section{Twin analyses}

The twin design seeks to partition variance in a phenotype into three components: additive genetic influences (A); shared environmental influences (C), which are common to both twins in a pair and cause similarity between them; and nonshared environmental influences (E), which are unique to each twin and create differences between them. These parameters are estimated on the basis of the phenotypic resemblance of monozygotic (MZ) twins, who share all their segregating DNA code, and dizygotic (DZ) twins, who share on average $50 \%$ of their segregating DNA code.

Cross-twin correlations were used to establish the phenotypic similarity of MZ and DZ twins. These correlations, estimated from a saturated model of the observed data, were obtained separately for $\mathrm{MZ}$ and $\mathrm{DZ}$ twins, and involved correlating one twin's score on a 
trait (e.g. autistic traits) with their co-twin's score on the same trait. One can then gain an indication of the extent of $\mathrm{A}, \mathrm{C}$, and $\mathrm{E}$ influences by examining the $\mathrm{MZ}$ and $\mathrm{DZ}$ cross-twin correlations. Table 1 shows how the pattern of cross-twin correlations can be interpreted.

The multivariate twin design allows one to investigate the covariance between multiple traits by dividing the correlation between them into A, C, and E components. Cross-trait cross-twin correlations were estimated as a starting point and involved correlating one twin's autistic traits with their co-twin's psychotic experiences score. Again derived separately for MZ and DZ twins, these correlations can be used to obtain an initial indication of the extent to which $\mathrm{A}, \mathrm{C}$, and $\mathrm{E}$ influence the covariance between two traits. Table 1 shows how cross-trait crosstwin correlations can be interpreted.

Structural equation twin-model fitting formally estimated A, C, and E. Cholesky decompositions were fitted to measures displaying sufficient covariance with one another $\left(r_{\mathrm{ph}}>.20\right.$ at age 16). These decompositions estimated the degree to which the causes of autistic traits could account for psychotic experiences. In Fig. 1, the pathways from latent variables (enclosed in circles) $A_{1}$, $C_{1}, E_{1}, A_{2}, C_{2}, E_{2}, A_{3}, C_{3}, E_{3}, A_{4}, C_{4}$, and $E_{4}$ to SPEQ subscales at 16 years denote the extent to which the causes of autistic traits influence psychotic experiences. In squaring these estimates, one can derive the proportions of variance explained. $A_{5}, C_{5}$, and $E_{5}$ represent residual variance in SPEQ scores that is unique to psychotic experiences.

In the first instance, all estimates were free to differ by sex (quantitative sex limitation), and were then equated across sexes to test the significance of any sex differences. Parameters were then successively dropped from the ACE Cholesky decomposition by fixing them to 0 . The fit of the Cholesky decompositions was compared against a baseline-saturated model of observed means and variances. The fit of each model was summarised by the fit statistic which was -2 times the log likelihood of the data $(-2 \mathrm{LL})$; differences in $-2 \mathrm{LL}$ between two models are $X^{2}$ distributed, with degrees of freedom equivalent to the difference in number of parameters. Significant $\chi^{2}$ results suggest a model is a significantly poorer fit than the saturated model. Model fit was further assessed using Akaike's Information Criteria (AIC) and Bayesian Information Criteria (BIC). Best-fitting models were selected based on the most negative BIC value.

Effects of two potential confounders were then tested: general cognitive ability and internalising traits. A composite g-score was computed when twins were aged 7 , 12, 14, and 16 years [41]. At age 7, twins completed three measures from the Wechsler Intelligence Test for Children (WISC [42]): picture completion, vocabulary, and similarities. Twins also completed the McCarthy Conceptual Groups test [43]. At age 12, twins completed the WISC vocabulary, picture completion, and general knowledge tests [42] as well as the Raven's Standard Progressive Matrices [44]. At ages 14 and 16, the Raven's Standard Progressive Matrices and WISC vocabulary tests were administered. At each age, scores on all completed measures were standardised; g-score was calculated as the average of the standardised test scores at each age.

Internalising traits were a second potential confounder. They were assessed using two self-report measures, the Childhood Anxiety Sensitivity Index [45] and Short Moods and Feelings Questionnaire [46]. Analyses




were repeated with each of these confounders regressed out of all measures.

\section{Results}

\section{Phenotypic analyses}

Descriptive statistics are in Table 2 and are shown by sex and zygosity in Additional file 2. Table 2 shows $r_{\mathrm{ph}}$ estimates; at age 16 years, the highest estimates were between autistic traits and negative symptoms $\left(r_{\mathrm{ph}}=.47\right.$, $p<.01)$, and autistic traits and anhedonia $\left(r_{\mathrm{ph}}=.30\right.$, $p<.01) . \mathrm{r}_{\mathrm{ph}}$ showed a trend for increasing with age; $r_{\mathrm{ph}}$ between autistic traits and negative symptoms increased from .27 at age 8 years to .47 at age 16 years, and from .14 to .30 for anhedonia. All other estimates were modest (.00-.21); therefore, only negative symptoms and anhedonia were included in twin-model fitting.

Cross-sectionally, negative symptoms showed stronger associations with attention switching $\left(r_{\mathrm{ph}}=.45, p<.01\right)$ and social difficulties $\left(r_{\mathrm{ph}}=.44, p<.01\right)$ than the other AQ subscales, as did anhedonia $\left(r_{\mathrm{ph}}=.25-.37\right)$. A full table of $r_{\text {ph }}$ between psychotic experiences and the autistic trait subscales is shown in Additional file 3.

\section{Twin analyses}

\section{Twin correlations}

Twin correlations are shown in Table 3. For autistic traits, MZ cross-twin correlations were higher than DZ estimates, suggesting A. MZ estimates were less than 1, suggesting E, while DZ estimates all exceeded half the MZ estimates, implicating C. For anhedonia and negative symptoms, MZ cross-twin correlations were higher than DZ statistics, implicating A. MZ estimates were all below 1, suggesting $\mathrm{E}$. In all instances, the MZ crosstrait cross-twin correlations between negative symptoms and autistic traits, and anhedonia and autistic traits were higher than the DZ estimates. This suggests that genetic

Table 2 Sample size, descriptive statistics, and phenotypic correlations

\begin{tabular}{|c|c|c|c|c|c|c|}
\hline \multicolumn{7}{|l|}{ Sample sizes } \\
\hline Age & $N$ families contacted & MZM & DZM & MZF & DZF & DZOS \\
\hline 8 years & 13,941 & 858 & 804 & 1046 & 874 & 1659 \\
\hline 12 years & 8438 & 814 & 775 & 1050 & 895 & 1601 \\
\hline 14 years & 11,118 & 529 & 463 & 662 & 548 & 911 \\
\hline 16 years & 10,874 & 738 & 686 & 1043 & 905 & 1560 \\
\hline \multicolumn{7}{|l|}{ Measure descriptive statistics } \\
\hline Measure & Cronbach's a & \multicolumn{2}{|c|}{ Possible range of scores } & \multicolumn{2}{|c|}{$\bar{x}(S D)$} & Skew \\
\hline CAST age 8 & 0.71 & \multicolumn{2}{|l|}{$0-31$} & \multicolumn{2}{|c|}{$4.85(3.09)$} & $0.99(-0.64)$ \\
\hline CAST age 12 & 0.73 & \multicolumn{2}{|l|}{$0-30$} & \multicolumn{2}{|c|}{$4.62(3.15)$} & $1.24(-0.48)$ \\
\hline AQ age 14 & 0.80 & \multicolumn{2}{|l|}{$0-87$} & \multicolumn{2}{|c|}{$36.78(12.16)$} & $0.24(0.21)$ \\
\hline AQ age 16 & 0.80 & \multicolumn{2}{|l|}{$0-80$} & \multicolumn{2}{|c|}{$24.04(10.62)$} & $0.39(0.36)$ \\
\hline SPEQ paranoia & 0.93 & \multicolumn{2}{|l|}{$0-72$} & \multicolumn{2}{|c|}{$12.17(10.62)$} & $1.56(-0.61)$ \\
\hline SPEQ hallucinations & 0.88 & \multicolumn{2}{|l|}{$0-45$} & \multicolumn{2}{|c|}{$4.66(6.01)$} & $2.08(0.22)$ \\
\hline SPEQ cognitive disorganisation & 0.77 & \multicolumn{2}{|l|}{$0-11$} & \multicolumn{2}{|c|}{$3.96(2.85)$} & $0.44(-0.63)$ \\
\hline SPEQ grandiosity & 0.86 & \multicolumn{2}{|l|}{$0-24$} & \multicolumn{2}{|c|}{$5.32(4.43)$} & $1.19(-0.41)$ \\
\hline SPEQ anhedonia & 0.78 & \multicolumn{2}{|l|}{$0-50$} & \multicolumn{2}{|c|}{$16.36(7.93)$} & $1.14(0.17)$ \\
\hline SPEQ negative symptoms & 0.86 & \multicolumn{2}{|l|}{$0-27$} & \multicolumn{2}{|c|}{$2.63(3.63)$} & $2.32(0.45)$ \\
\hline \multicolumn{7}{|l|}{ Phenotypic correlations } \\
\hline & Autistic traits age 8 & \multicolumn{2}{|c|}{ Autistic traits age 12} & \multicolumn{2}{|c|}{ Autistic traits age 14} & Autistic traits age 16 \\
\hline Paranoia & .02 (ns) & \multicolumn{2}{|l|}{$.06^{*}$} & \multicolumn{2}{|l|}{$.07^{* *}$} & $.10^{* *}$ \\
\hline Hallucinations & $.07^{* *}$ & \multicolumn{2}{|l|}{$.10^{* *}$} & \multicolumn{2}{|l|}{$.13^{* *}$} & $.14^{* *}$ \\
\hline Cognitive disorganisation & $.08^{* *}$ & \multicolumn{2}{|l|}{$.12^{* *}$} & \multicolumn{2}{|l|}{$.17^{* *}$} & $.21^{* *}$ \\
\hline Grandiosity & $.04^{*}$ & $.08^{* *}$ & & $.04(n$ & & .00 (ns) \\
\hline Anhedonia & $.14^{* *}$ & $.18^{* *}$ & & $.24^{* *}$ & & $.30^{* *}$ \\
\hline Negative symptoms & $.27^{* *}$ & $.32^{* *}$ & & $.40^{* *}$ & & $.47^{* *}$ \\
\hline
\end{tabular}

All means are given for untransformed scores; skew values are shown for the untransformed measures, and then the standardised residuals used in analyses in parentheses. All Ns are number of twin pairs with data available at each age SPEQ Specific Psychotic Experiences Questionnaire, CAST Childhood Autism Spectrum Test, AQ Autism Spectrum Quotient, ns nonsignificant ${ }^{*} p<.05 ;{ }^{* *} p<.01$ 
Table 3 Twin correlations

\begin{tabular}{|c|c|c|c|c|c|c|c|c|c|c|}
\hline \multicolumn{11}{|l|}{ Cross-twin correlations } \\
\hline & \multicolumn{2}{|l|}{ MZM } & \multicolumn{2}{|l|}{ DZM } & \multicolumn{2}{|l|}{ MZF } & \multicolumn{2}{|l|}{$\mathrm{DZF}$} & \multicolumn{2}{|l|}{ DZOS } \\
\hline & Estimate & $95 \% \mathrm{Cl}$ & Estimate & $95 \% \mathrm{Cl}$ & Estimate & $95 \% \mathrm{Cl}$ & Estimate & $95 \% \mathrm{Cl}$ & Estimate & $95 \% \mathrm{Cl}$ \\
\hline Autistic traits age 8 & .83 & $.81 / .85$ & .37 & $.35 / .39$ & .79 & $.76 / .82$ & .45 & $.40 / .49$ & .48 & $.44 / .49$ \\
\hline Autistic traits age 12 & .79 & $.77 / .82$ & .32 & $.29 / .35$ & .76 & $.73 / .79$ & .51 & $.47 / .55$ & .41 & $.38 / .45$ \\
\hline Autistic traits age 14 & .91 & $.88 / .93$ & .54 & $.51 / .56$ & .88 & $.85 / .89$ & .62 & $.60 / .66$ & .51 & $.47 / .55$ \\
\hline Autistic traits age 16 & .92 & $.90 / .95$ & .51 & $.48 / .55$ & .82 & $.80 / .87$ & .58 & $.55 / .61$ & .46 & $.44 / .50$ \\
\hline Anhedonia & .37 & $.31 / .39$ & .19 & $.18 / .22$ & .45 & $.42 / .46$ & .17 & $.13 / .19$ & .14 & $.12 / .15$ \\
\hline Negative symptoms & .80 & $.78 / .82$ & .59 & $.55 / .60$ & .81 & $.78 / .82$ & .52 & $.47 / .54$ & .47 & $.44 / .48$ \\
\hline \multicolumn{11}{|l|}{ Cross-trait cross-twin correlations } \\
\hline & \multicolumn{2}{|l|}{ MZM } & \multicolumn{2}{|l|}{ DZM } & \multicolumn{2}{|l|}{ MZF } & \multicolumn{2}{|l|}{ DZF } & \multicolumn{2}{|l|}{ DZOS } \\
\hline & Estimate & $95 \% \mathrm{Cl}$ & Estimate & $95 \% \mathrm{Cl}$ & Estimate & $95 \% \mathrm{Cl}$ & Estimate & $95 \% \mathrm{Cl}$ & Estimate & $95 \% \mathrm{Cl}$ \\
\hline Autistic traits age 8 -anhedonia & .18 & $.15 / .22$ & .07 & $.03 / .10$ & .22 & $.19 / .23$ & .03 & $.01 / .05$ & .08 & $.05 / .09$ \\
\hline Autistic traits age 12-anhedonia & .20 & $.17 / .23$ & .00 & $-.05 / .04$ & .23 & $.19 / .26$ & .07 & $.03 / .09$ & .11 & $.05 / .13$ \\
\hline Autistic traits age $14-$ anhedonia & .24 & $.15 / .27$ & .16 & $.09 / .20$ & .32 & $.21 / .34$ & .12 & $.08 / .15$ & .14 & $.04 / .17$ \\
\hline Autistic traits age 16 -anhedonia & .28 & $.24 / .29$ & .15 & $.09 / .17$ & .31 & $.29 / .33$ & .11 & $.06 / .12$ & .11 & $.09 / .13$ \\
\hline Autistic traits age 8 -negative symptoms & .22 & $.19 / .23$ & .18 & $.15 / .19$ & .24 & $.21 / .25$ & .19 & $.15 / .22$ & .20 & $.18 / .22$ \\
\hline Autistic traits age 12-negative symptoms & .34 & $.31 / .35$ & .24 & $.22 / .25$ & .29 & $.27 / .31$ & .19 & $.17 / .22$ & .19 & $.15 / .22$ \\
\hline Autistic traits age 14-negative symptoms & .37 & $.31 / .44$ & .30 & $.22 / .36$ & .32 & $.23 / .36$ & .25 & $.18 / .29$ & .24 & $.21 / .27$ \\
\hline Autistic traits age 16 -negative symptoms & .43 & $.42 / .45$ & .31 & $.29 / .34$ & .36 & $.34 / .38$ & .30 & $.28 / .36$ & .21 & $.19 / .23$ \\
\hline
\end{tabular}

MZM monozygotic male, DZM dizygotic male, MZF monozygotic female, DZF dizygotic female, DZOS dizygotic opposite sex

factors played a role in the covariance between autistic traits and negative symptoms, and autistic traits and anhedonia.

\section{Twin-model fitting}

Five-variable Cholesky decompositions were fitted to (1) autistic traits and anhedonia, and (2) autistic traits and negative symptoms. Model-fit statistics and parameter estimates are shown in Table 4.

Autistic traits and anhedonia An ACE decomposition with no sex differences best fit autistic traits and anhedonia. Paths $\mathrm{A}_{1}-\mathrm{A}_{4}, \mathrm{C}_{1}-\mathrm{C}_{4}$, and $\mathrm{E}_{1}-\mathrm{E}_{4}$ in Fig. 1 denote the genetic and environmental influences on childhood autistic traits that also influence anhedonia at age 16 years. Summing these paths gives the total proportion of variance in anhedonia explained by autistic traits. In total, $10 \%$ of the variance in anhedonia was explained by autistic traits. Of this variance, half (5\%) was explained by A influences on autistic traits, $3 \%$ was due to $\mathrm{C}$ influences shared with autistic traits, and $2 \%$ was due to $\mathrm{E}$. Of the $90 \%$ of variance in anhedonia that was independent of autistic traits, $32 \%$ was explained by A and $58 \%$ by E. Anhedonia was $37 \%$ heritable, yet only $5 / 37=13.5 \%$ of this heritability was due to genetic influences shared with childhood autistic traits.
Autistic traits and negative symptoms An ACE Cholesky composition with no sex differences best fit autistic traits and negative symptoms. $\mathrm{A}, \mathrm{C}$, and $\mathrm{E}$ influences on autistic traits between ages 8-16 years $\left(\mathrm{A}_{1}-\mathrm{A}_{4}, \mathrm{C}_{1}-\mathrm{C}_{4}\right.$, and $\left.\mathrm{E}_{1}-\mathrm{E}_{4}\right)$ accounted for $31 \%$ of the variance in negative symptoms. Of this shared variance, $12 \%$ was explained by A influences on autistic traits, $16 \%$ was due to $C$, and $3 \%$ was due to $E$. Of the $69 \%$ variance in negative symptoms that was independent of autistic traits, most ( $45 \%$ ) was explained by $\mathrm{A}$, while $\mathrm{C}$ and $\mathrm{E}$ comprised $10 \%$ and $14 \%$, respectively. Negative symptoms were $57 \%$ heritable, yet only $12 / 57=21 \%$ of this heritability was due to genetic influences shared with childhood autistic traits.

Controlling for confounders Controlling for g-score or internalising traits resulted in nonsignificant reductions in the phenotypic correlations. Consequently, we did not repeat twin-model fitting on these adjusted scores (contact the first author for details).

\section{Individuals with ASC}

Mean standardised SPEQ scores for participants with ASC are shown in Fig. 2. Mean scores for controls were 0 owing to the standardisation of scores. Individuals with ASC displayed significantly higher scores than controls for cognitive disorganisation $\left(t_{24.54}=-6.68, p<.001\right.$, 
Table 4 Twin-model fit statistics and parameter estimates

\begin{tabular}{|c|c|c|c|c|c|c|c|c|c|c|c|c|}
\hline \multirow{2}{*}{$\overline{\text { Model }}$} & \multirow{2}{*}{$-2 \mathrm{LL}$} & \multirow[b]{2}{*}{$d f$} & \multirow[b]{2}{*}{ Par. } & \multirow[b]{2}{*}{$\mathrm{BIC}$} & \multicolumn{4}{|c|}{ Comparative fit with saturated model } & \multicolumn{4}{|c|}{ Comparative fit with full ACE Cholesky } \\
\hline & & & & & $\Delta x^{2}$ & $\Delta \mathrm{df}$ & $p$ & AIC & $\Delta x^{2}$ & $\Delta \mathrm{df}$ & $p$ & AIC \\
\hline \multicolumn{13}{|c|}{$\begin{array}{l}\text { Autistic traits and anhedonia } \\
\text { (five-variable decompositions) }\end{array}$} \\
\hline Saturated & $63,287.21$ & 27,375 & 260 & -158948.70 & - & - & - & - & - & - & - & - \\
\hline ACE sex diff. & $63,489.32$ & 27,535 & 100 & -160045.50 & 202.11 & 160 & $<.05$ & -117.89 & - & - & - & - \\
\hline ACE const. ${ }^{a}$ & $63,619.71$ & 27,580 & 55 & -160280.40 & 332.50 & 205 & $<.001$ & -77.50 & 130.39 & 45 & $<.001$ & 40.39 \\
\hline AE const. & $63,784.42$ & 27,595 & 40 & -160237.50 & 497.20 & 220 & $<.001$ & 57.20 & 164.71 & 15 & $<.001$ & 134.71 \\
\hline CE const. & $64,974.16$ & 27,595 & 40 & -159047.80 & 1686.94 & 220 & $<.001$ & 1246.94 & 1345.45 & 15 & $<.001$ & 1324.45 \\
\hline E const. & $71,621.98$ & 27,610 & 25 & -152521.80 & 8334.77 & 235 & $<.001$ & 7864.77 & 8002.27 & 30 & $<.001$ & 7942.27 \\
\hline \multicolumn{13}{|c|}{$\begin{array}{l}\text { Autistic traits and parent-rated negative } \\
\text { symptoms (five-variable decompositions) }\end{array}$} \\
\hline Saturated & $60,014.32$ & 27,393 & 260 & -162367.70 & - & - & - & - & - & - & - & - \\
\hline ACE sex diff. & $60,189.54$ & 27,553 & 100 & -163491.40 & 175.22 & 160 & .19 & -144.78 & - & - & - & - \\
\hline ACE const. ${ }^{a}$ & $60,319.68$ & 27,598 & 55 & -163726.60 & 305.35 & 205 & $<.001$ & -104.65 & 130.13 & 45 & $<.001$ & 40.13 \\
\hline $\mathrm{AE}$ & $60,542.65$ & 27,613 & 40 & -163625.40 & 528.33 & 220 & $<.001$ & -7.02 & 222.98 & 15 & $<.001$ & 192.98 \\
\hline CE & $61,994.75$ & 27,613 & 40 & -162173.31 & 1980.42 & 220 & $<.001$ & 1540.42 & 1675.07 & 15 & $<.001$ & 1645.07 \\
\hline E & $70,414.98$ & 27,628 & 25 & -153874.80 & 10400.66 & 235 & $<.001$ & 9930.66 & 10095.31 & 30 & $<.001$ & 10035.31 \\
\hline
\end{tabular}

Parameter estimates from best-fitting decompositions

Autistic traits age $8 \quad$ Autistic traits age 12
$\begin{array}{lll}A_{1} & C_{1} & E_{1}\end{array}$
$\mathrm{A}_{2}$
$\mathrm{C}_{2}$
$E_{2}$
$\begin{array}{llllllllllllllll}\text { Anhedonia } & .02(.00 / .05) & .01(.00 / .05) & .00(-.01 / .01) & .00(.00 / .03) & .01(.00 / .04) & .00(-.01 / .01) & .02(.00 / .05) & .01(.00 / .04) & .00(.00 / .01) & .01 & .00 & .02 & .02 & .00 & .52\end{array}$

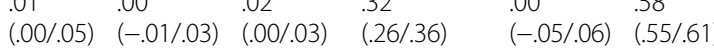

Autistic traits age 14

Autistic traits age 16

Variance specific to psychotic experiences Total
.03
.01
.03
Neg sym $\quad .02(.00 / .04) \quad .10(.04 / .22) \quad .00(.00 / .01)$
Total $\quad .12$
$.01(.00 / .03) \quad .06(.01 / .17) \quad .00(.00 / .01) \quad .05(.03 / .10) \quad .00$
.03
.90
$.12-07$
.06
$\begin{array}{lllll}.00 & .01(.00 / .01) & .04 & .00 & .02 \\ (-.01 / .01) & & (.01 / .07) & (-.02 / .02) & (.01 / .03)\end{array}$
$.45(.40 / .50) \quad .10 \quad .14$
$07 \quad .06$
.06
.69

$A E$, $C E$, and $E$ models are submodels within the $A C E$ model that fit best. $A_{1}-A_{4}, C_{1}-C_{4}$, and $E_{1}-E_{4}$ are the paths shown in red in Fig. 1, and represent the influence of genetic and environmental causes of autistic traits on psychotic experiences, $A_{5}, C_{5}$, and $E_{5}$ are residual pathways (shown in blue in Fig. 1), which represent the genetic and environmental influences on psychotic experiences that are independent of autistic traits Saturated saturated model of means and covariance, ACE sex diff. full ACE Cholesky decomposition, which included quantitative sex differences for all parameters, ACE const. constrained ACE Cholesky decomposition, with parameters fixed to be equal across sexes, $-2 L L$ fit statistic, $d f$ degrees of freedom, Par. parameters, AIC Akaike's Information Criteria, BIC Bayesian Information Criteria, $A$ additive genetic influences, $C$ shared environmental influences, $E$ nonshared environmental influences

andicates best-fitting model 




$r=.80)$, anhedonia $\left(t_{24.20}=-2.55, p<.05, r=.46\right)$, and negative symptoms $\left(t_{24.20}=-2.55, p<.05, r=.80\right)$.

\section{Family history of psychotic disorders}

Mean AQ scores at age 16 were virtually identical across individuals with a first-degree relative with a psychotic disorder $(\bar{x}=24.76[\mathrm{SD}=11.68])$ and those without $(\bar{x}=$ 24.14 [SD $=10.81])$. Mean scores did not differ significantly across individuals with and without a family history of a psychotic disorder, $t_{489.53}=-1.04, p=.30$.

\section{Discussion}

Childhood autistic traits, and diagnosed ASC, were associated with negative psychotic experiences in adolescence, but not positive or cognitive psychotic experiences. As expected, twin modelling revealed a degree of shared genetic and environmental influences between these traits, yet this was modest. The majority of variance in adolescent negative symptoms was independent of autistic traits. In addition, there was no indication of an elevation in autistic traits for individuals with a family history of psychotic disorders. Thus, our results imply a limited association between autistic traits and psychotic experiences. These findings could not be accounted for by general cognitive ability or internalising traits.

Our findings concur with two previous studies in showing that autistic traits are linked with negative symptoms, in particular [13, 14], yet associations with positive and cognitive psychotic experiences were weaker. These findings seemingly contradict two community-based studies $[10,11]$. One possibility is that the use of different raters for autistic traits and these psychotic experiences in our study dampened the associations between them. This, however, is unable to explain why the association with 
negative symptoms held across both parent and self reports. It is notable that, while the association was weaker than with negative symptoms, cognitive psychotic experiences still showed a stronger correlation with autistic traits than positive symptoms in our study. It may, therefore, be that the previous findings were being driven by cognitive symptoms.

One of the core questions posed in this study was whether or not autistic traits would share genetic causes with psychotic experiences. Could the genetic variants associated with both ASC and schizophrenia, such as calcium-signalling genes [19] and 16p11.2 duplications $[47,48]$, mean that genetic factors might also play a role in influencing the co-occurrence of autistic traits and psychotic experiences in the general population? The genetic influences on autistic traits accounted for some variance in negative symptoms; however, this was only a small proportion of variance. More importantly, autistic traits were not as strongly linked with positive symptoms, such as paranoia, emphasising the possibility that any genetic associations between autistic traits and psychotic experiences are constrained to negative psychotic experiences. As such, we do not predict that studies of the genetic basis of psychotic experiences are likely to prove informative when attempting to understand the aetiology of ASC.

In light of recent findings [19, 47, 48], some have argued that clinical ASC and schizophrenia are part of a continuum of neurodevelopmental causality [49]. Indeed, there is evidence of some genetic and environmental overlap across autistic traits and traits of other conditions, including ADHD and anxiety [50,51]. The present findings can be taken as partial support to the idea of a continuum of neurodevelopmental conditions, with two key caveats. First, the relationship between autistic traits and psychotic experiences was limited to negative psychotic experiences. Second, the majority of the genetic and environmental influences on psychotic experiences were distinct from autistic traits, tallying with SNP-based evidence reporting low genetic correlations between ASC and schizophrenia [19]. As such, these findings converge to suggest that while there is some overlap between these phenotypes, they are largely separate, with low genetic overlap.

Given the modest role played by shared environment in autistic traits [22] and psychotic experiences [21], it is surprising that such influences on autistic traits accounted for some variance in psychotic experiences. The net influence of shared environment on anhedonia was weak, however. The use of the same rater for negative symptoms and autistic traits may also have inflated the role of shared environment in their covariance.

This study was not free from limitations. As with all studies of co-occurrence of different traits that rely on questionnaire data, there is some risk that items can be interpreted in different ways by different people and as such overlap could be for heterogeneous reasons. However, all the evidence here suggests that each of our measures is capturing what they aim to capture, and thus, any overlap is due to true co-occurrence of psychotic experiences and autistic traits in individuals.

A consideration is whether or not the association between autistic traits, anhedonia and negative symptoms, was driven by phenotypic similarity of the items. In the first instance, we removed an ostensibly overlapping item from the negative symptoms subscale of the SPEQ ('Has few or no friends'). Additionally, the correlations between scores on these measures were not total, indicating a degree of independence between the measures. Finally, our measures of autistic traits and psychotic experiences both have good construct and content validity, as outlined in the Methods" section.

Some may query the use of self-report measures in individuals with ASC, but it needs to be noted that the elevation in negative symptoms in individuals with ASC was evident not only for self-reported anhedonia, but also for parent-rated negative symptoms. It is worth noting, however, that autistic traits were rated by parents only. Future research should endeavour to obtain ratings of autistic traits from multiple informants, for example teachers in addition to parents. Finally, the use of a large sample, a requisite for twin-model fitting, necessitated the use of questionnaire measures over in-depth assessments. As such, the sample comprised not only individuals displaying very high scores on the measures, but also a considerable number of individuals displaying lower scores, resulting in skewed measures. This, however, is to be expected in large, community-based studies of psychopathology.

While we had longitudinal data on autistic traits, we did not have longitudinal data on psychotic experiences. Longitudinal cross-lagged twin models can test whether one phenotype directly leads to another e.g. [51], but the absence of data on psychotic experiences at multiple ages in our sample meant that this was not possible in our study. Nevertheless, this would represent an important future direction for research.

One might question whether these twin findings generalise to singletons. Neither autistic traits [52] nor psychotic experiences [8] appear elevated in twins relative to singletons. Studies of singletons have also reported that psychotic experiences are elevated in individuals with ASC [10-14].

\section{Conclusions}

These findings demonstrate that autistic traits and ASC were linked with negative symptoms in adolescence, such as anhedonia and affective flattening, but less so with cognitive or positive psychotic experiences. In 
clinics, therefore, one might expect to see individuals with ASC present with more negative symptoms, rather than positive symptoms, such as hallucinations and paranoia. Despite the co-occurrence of autistic traits and ASC with negative symptoms, however, genetic overlap between them was low. There remains a considerable amount of work to be done to fully unravel the genetic basis of autistic traits and psychotic experiences. As we learn more about the causes of these phenotypes, on the basis of the present findings, we do not predict that the same genetic variants will be identified as associated with autistic traits and psychotic experiences.

\section{Additional files}

\section{Additional file 1: Characteristics of participating and}

nonparticipating families in LEAP. Characteristics of participants who returned LEAP questionnaires compared with those who did not, including sex, zygosity, ethnicity, and maternal education level.

Additional file 2: Descriptive statistics on all measures by sex and zygosity. Mean scores and standard deviations on measures of autistic traits at all ages and the six psychotic experience subscales, including ANOVAs.

Additional file 3: Phenotypic correlations between autistic trait subscales and SPEQ subscales. Correlations between subscales of the autistic trait measures at all ages and the six psychotic experiences subscales.

\section{Abbreviations}

A: additive genetic influences; AIC: Akaike's Information Criteria; BIC: Bayesian Information Criteria; C: shared environmental influences; E: nonshared environmental influences; $r_{\mathrm{ph}}$ : phenotypic correlation.

\section{Competing interests}

The authors declare that they have no competing interests.

\section{Authors' contributions}

MJT designed the study, conducted the statistical analyses, and drafted the manuscript; EBR assisted in the design of the study; FH and PB oversaw the collection of ADOS and ADI-R data; DF assisted in the design of the LEAP study; AR assisted in the design of this study and designed the LEAP study. All authors assisted in the preparation of the manuscript. All authors read and approved the manuscript.

\section{Acknowledgements}

We thank the TEDS participants and team, including Andrew McMillan, Francesca Lewis, Louise Webster, Neil Harvey, Rachel Ogden, Peter McGuffin, Robert Plomin, Emma Colvert (all King's College London), and the Social Relationships Study team. This research was funded by the Medical Research Council [G100559 to Angelica Ronald; G0500870 to Francesca Happé and Patrick Bolton; G0901245, and previously G0500079, to Robert Plomin]. Patrick Bolton was supported by a NIHR Senior Investigator Award and the South London and Maudsley Hospital Biomedical Research Centre in Mental Health. Daniel Freeman is supported by an MRC Senior Clinical Fellowship and is a member of the Wellcome Trust Oxford Sleep and Circadian Neuroscience Institute (SCNi).

\section{Author details}

${ }^{1}$ Centre for Brain and Cognitive Development, Department of Psychological Sciences, Birkbeck, University of London, London, UK. ${ }^{2}$ Analytic and Translational Genetics Unit, Department of Medicine, Massachusetts General Hospital and Harvard Medical School, Boston, MA, USA. ${ }^{3}$ Stanley Center for Psychiatric Research and Program in Medical and Population Genetics, Broad Institute of Harvard and MIT, Cambridge, MA, USA. ${ }^{4}$ MRC Social, Genetic and Developmental Psychiatry Centre, Institute of Psychiatry, Psychology \&
Neuroscience, King's College, London, UK. ${ }^{5}$ Department of Child Psychiatry and MRC SGDP Centre, Institute of Psychiatry, Psychology \& Neuroscience, King's College, London, UK. ${ }^{6}$ Department of Psychiatry, University of Oxford, Oxford, UK.

Received: 19 February 2015 Accepted: 8 July 2015

Published online: 22 July 2015

\section{References}

1. American Psychiatric Association. Diagnostic and statistical manual of mental disorders. 5th ed. Washington, DC: American Psychiatric Association; 2013.

2. Howlin P. Autism and Asperger Syndrome: preparing for adulthood London: Routledge; 2004.

3. Leyfer OT, Folstein SE, Bacalman S, Davis NO, Dinh E, Morgan J, et al. Comorbid psychiatric disorders in children with autism: interview development and rates of disorder. J Autism Dev Disord. 2006;36:849-61.

4. Levy SE, Giarelli E, Lee L, Schieve LA, Kirby RS, Cunniff C, et al. Autism spectrum disorder and co-occurring developmental, psychiatric, and medical conditions among children in multiple populations of the United States. J Dev Behav Pediatr. 2010;31:267-75.

5. Joshi G, Wozniak J, Petty C, Martelon MK, Fried R, Bolfek A, et al. Psychiatric comorbidity and functioning in a clinically referred population of adults with autism spectrum disorders: a comparative study. J Autism Dev Disord. 2013;43:1314-25

6. Laursen TM, Munk-Olsen T, Norderntoft M, Bo MP. A comparison of selected risk factors for unipolar depressive disorder, bipolar affective disorder, schizoaffective disorder, and schizophrenia from a Danish population-based cohort. J Clin Psychiatry. 2007;68:1673-81.

7. Poulton R, Caspi A, Moffitt TE, Cannon M, Murray R, Harrington H. Children's self-reported psychotic symptoms and adult schizophreniform disorder: a 15-year longitudinal study. Arch Gen Psychiatry. 2000;57:1053-8.

8. Ronald A, Sieradzka D, Cardno AG, Haworth CMA, McGuire P, Freeman D. Characterization of psychotic experiences in adolescence using the Specific psychotic experiences questionnaire: findings from a study of 5000 16-year-olds. Schizophr Bull. 2014;40:868-77.

9. Dominguez MD, Wichers M, Lieb R, Wittchen HU, van Os J. Evidence that onset of clinical psychosis is an outcome of progressively more persistent subclinical psychotic experiences: an 8-year cohort study. Schizophr Bull. 2011;37:84-93

10. Sullivan S, Rai D, Golding J, Zammit S, Steer C. The association between autism spectrum disorder and psychotic experiences in the Avon longitudinal study of parents and children (ALSPAC). J Am Acad Child Adolesc Psychiatry. 2013;52:806-14.

11. Bevan Jones R, Thapar A, Lewis A, Zammit S. The association between early autistic traits and psychotic experiences in adolescence. Schizophr Res. 2012;135:164-9.

12. de Bruin El, Ferdinand RF, Meester S, de Nijs PFA, Verheij F. High rates of psychiatric co-morbidity in PDD-NOS. J Autism Dev Disord. 2007;37:877-86.

13. Gadow KD. Schizophrenia spectrum and attention-deficit/hyperactivity disorder symptoms in autism spectrum disorder and controls. J Am Acad Child Adolesc Psychiatry. 2012;51:1076-84

14. Gadow KD. Association of schizophrenia spectrum and autism spectrum disorder (ASD) symptoms in children with ASD and clinic controls. Res Dev Disabil. 2013;34:1289-99.

15. Brett C, Heriot-Maitland C, McGuire P, Peters E. Predictors of distress associated with psychotic-like anomalous experiences in clinical and nonclinical populations. Br J Clin Psychol. 2014;53:213-27.

16. Kelleher I, Devlin N, Wigman JT, Kehoe A, Murtagh A, Fitzpatrick C, et al. Psychotic experiences in a mental health clinic sample: implications for suicidality, multimorbidity and functioning. Psychol Med. 2014:44:1615-24

17. Kelleher I, Cannon M. Psychotic-like experiences in the general population: characterizing a high-risk group for psychosis. Psychol Med. 2011;41:1-6.

18. Sullivan PF, Magnusson C, Reichenberg A, Boman M, Dalman C, Davidson $\mathrm{M}$, et al. Family history of schizophrenia and bipolar disorder as risk factors for autism. Arch Gen Psychiatry. 2012;69:1099-103.

19. Cross-Disorder Group of the Psychiatric Genomics Consortium. Identification of risk loci with shared effects on five major psychiatric disorders: a genome-wide analysis. Lancet. 2013;381:1371-9. 
20. Purcell SM, Moran JL, Fromer M, Ruderfer D, Solovieff N, Roussos P, et al. A polygenic burden of rare disruptive mutations in schizophrenia. Nature. 2014,506:185-90

21. Zavos HMS, Freeman D, Haworth CMA, McGuire P, Plomin R, Cardno AG, et al. Consistent etiology of severe, frequent psychotic experiences and milder, less frequent manifestations: a twin study of specific psychotic experiences in adolescence. JAMA Psychiatry. 2014;71:1049-57.

22. Robinson EB, Koenen KC, McCormick MC, Munir K, Hallett V, Happé F, et al. Evidence that autistic traits show the same etiology in the genera population and the quantitative extremes (5\%, 2.5\%, and 1\%). Arch Gen Psychiatry. 2011;68:1113-21.

23. Wigman JT, Vollebergh WA, Raaijmajers QA, ledema J, van Dorsselaer S, Ormel J, et al. The structure of the extended psychosis phenotype in early adolescence: a cross-sample replication. Schizophr Bull. 2011;37:850-60.

24. Haworth CMA, Davis OSP, Plomin R. Twins Early Development Study (TEDS): a genetically sensitive investigation of cognitive and behavioral development from childhood to young adulthood. Twin Res Hum Genet. 2013:16:117-25.

25. Price TS, Freeman B, Craig I, Petrill SA, Ebersole L, Plomin R. Infant zygosity can be assigned by parental report questionnaire data. Twin Res. 2000;3:129-33.

26. Scott FJ, Baron-Cohen S, Bolton P, Brayne C. The CAST (Childhood Asperger Syndrome Test): preliminary development of a UK screen for mainstream primary-school-age children. Autism. 2002;6:9-31.

27. Williams J, Scott F, Stott C, Allison C, Bolton P, Baron-Cohen S, et al. The CAST (Childhood Asperger Syndrome Test): test accuracy. Autism. 2005:9:45-68.

28. Ronald A, Happé F, Bolton P, Butcher LM, Price TS, Wheelwright S, et al. Genetic heterogeneity between the three components of the autism spectrum: a twin study. J Am Acad Child Adolesc Psychiatry. 2006;45:691-9.

29. Baron-Cohen S, Wheelwright S, Skinner R, Martin J, Clubley E. The AutismSpectrum Quotient (AQ): evidence from asperger syndrome/highfunctioning autism, males and females, scientists and mathematicians. J Autism Dev Disord. 2001;31:5-17.

30. Baron-Cohen S, Hoekstra RA, Knickmeyer R, Wheelwright S. The AutismSpectrum Quotient (AQ) —adolescent version. J Autism Dev Disord. 2006:36:343-50.

31. Goodman R, Ford T, Richards H, Gatward R, Meltzer H. The development and well-being assessment: description and internal validation of an integrated assessment of child and adolescent psychopathology. J Child Psychol Psychiatry. 2000;41:645-55.

32. Dworzynski K, Happé F, Bolton P, Ronald A. Relationship between symptom domains in autism spectrum disorders: a population based twin study. $J$ Autism Dev Disord. 2009;39:1197-210.

33. Colvert E, Tick B, McEwen F, Ames C, Curran S, Woodhouse E, et al. Heritability of autism spectrum disorder in a UK twin sample. JAMA Psychiatry. 2015;72(5):415-23.

34. Lord C, Rutter M, Goode S, Heemsbergen J, Jordan H, Mawhood L, et al. Autism diagnostic observation schedule: a standardized observation of communicative and social behavior. J Autism Dev Disord. 1989;19:185-212.

35. Lord C, Rutter M, Le Couteur A. Autism diagnostic interview-revised: a revised version of a diagnostic interview for caregivers of individuals with possible pervasive developmental disorders. J Autism Dev Disord. 1994:24:659-85.

36. Blanchard JJ, Kring AM, Horan WP, Gur R. Toward the next generation of negative symptoms assessments: the collaboration to advance negative symptom assessment in Schizophrenia. Schizophr Bull. 2011;37:291-9.

37. Zammit S, Owen MJ, Evans J, Heron J, Lewis G. Cannabis, COMT and psychotic experiences. Br J Psychiatry. 2011;199:380-5.

38. McGue M, Bouchard TJ. Adjustment of twin data for the effects of sex and age. Behav Genet. 1984;14:325-43.

39. Boker SM, Neale MC, Maes HM, Wilde MJ, Spiegel M, Brick TR, et al OpenMx: an open source extended structural equation modeling framework. Richmond, VA: Psychometrika; 2011.

40. R Core Team. R: a language and environment for structural computing Vienna: R Foundation for Statistical Computing; 2013.

41. Davis OSP, Haworth CM, Plomin R. Learning abilities and disabilities: generalist genes in early adolescence. Cogn Neuropsychiatry. 2009;14:312-31.
42. Wechsler D. Weschler intelligence scale for children. 3rd ed. London: The Psychological Corporation; 1992.

43. McCarthy D. McCarthy scales of children's abilities. New York: The Psychological Corporation; 1972.

44. Raven JC, Court JH, Raven J. Manual for Raven's progressive matrices and vocabulary scales. Oxford: Oxford University Press; 1996.

45. Silverman WK, Fleisig W, Rabian B, Peterson RA. Childhood anxiety sensitivity index. J Clin Child Psychol. 1991;20:162-8.

46. Angold A, Costello EJ, Messer SC, Pickles A, Winder F, Silver D. The development of a short questionnaire for use in epidemiological studies of depression in children and adolescents. Int J Methods Psychiatric Res. 1995:5:1-12.

47. Steinberg S, de Jong S, Mattheisen M, Costas J, Demontis D, Jamain S, et al. Common variant at 16 p11.2 conferring risk of psychosis. Mol Psychiatry. 2014;19:108-14.

48. Crespi BJ, Crofts HJ. Association testing of copy number variants in schizophrenia and autism spectrum disorders. J Neurodev Disord. 2012;30:15.

49. Owen MJ. The origins of schizophrenia. Br J Psychiatry. 2012;201:162

50. Hallett V, Ronald A, Rijsdijk F, Happé F. Association of autistic-like and internalizing traits during childhood: a longitudinal twin study. Am J Psychiatry. 2010;167:809-17.

51. Taylor MJ, Charman T, Robinson EB, Plomin R, Happé F, Asherson A, et al. Developmental associations between traits of autism spectrum disorder and attention deficit hyperactivity disorder: a genetically-informative, longitudinal twin study. Psychol Med. 2013;43:1735-46.

52. Curran S, Dworzynski K, Happé F, Ronald A, Allison C, Baron-Cohen S, et al. No major effect of twinning on autistic traits. Autism Res. 2011:4:377-82.

\section{Submit your next manuscript to BioMed Central and take full advantage of:}

- Convenient online submission

- Thorough peer review

- No space constraints or color figure charges

- Immediate publication on acceptance

- Inclusion in PubMed, CAS, Scopus and Google Scholar

- Research which is freely available for redistribution 